November 1993

cond-mat/nnnmmyyy

\title{
NEW CRITICALITY OF 1D FERMIONS
}

\author{
Michael Lässig \\ Institut für Festkörperforschung \\ Forschungszentrum, 52425 Jülich, Germany \\ email:lassig@iff011.dnet.kfa-juelich.de
}

\begin{abstract}
One-dimensional massive quantum particles (or 1 + 1-dimensional random walks) with short-ranged multi-particle interactions are studied by exact renormalization group methods. With repulsive pair forces, such particles are known to scale as free fermions. With finite $m$-body forces $(m=3,4, \ldots)$, a critical instability is found, indicating the transition to a fermionic bound state. These unbinding transitions represent new universality classes of interacting fermions relevant to polymer and membrane systems. Implications for massless fermions, e.g. in the Hubbard model, are also noted.
\end{abstract}

PACS numbers: 5.70Jk, 5.40+j, 64.60Ak

to appear in Phys. Rev. Lett. 
Interacting quantum particles moving in one spatial dimension and imaginary time offer a unifying description of most 2D fluctuating systems. The trajectories of these particles represent (streched) polymers, domain walls or interfaces, steps on surfaces, magnetic flux lines, etc. Two ensembles have to be distinguished: (a) Vicinal surfaces [四] or systems at a 2D bulk critical point (e.g. Curie point, commensurate-to-incommensurate transition [2], surface reconstruction transition [3]) contain a finite density of such lines and are described by a massless quantum field theory which is generically isotropic and conformally invariant. (b) Systems with only a finite number of directed lines are ensembles of massive particles. Such systems may exhibit critical behavior at delocalization transitions between a low-temperature dense phase and a high-temperature dilute phase [ [

In the dense phase, the lines are bound to a bundle of transversal extension $\xi_{\perp}$. Their relative fluctuations are thus constrained; correlations in longitudinal direction decay on a scale $\xi_{\|}$. This phase is a bound state of the quantum particles. In the dilute phase, the lines fluctuate independently; the quantum particles are in a delocalized state. As the transition temperature is approached from below, the length scales $\xi_{\|}$and $\xi_{\perp}=\xi_{\|}^{\zeta}$ diverge. These transitions are generically anisotropic; the roughness exponent $\zeta$ equals $1 / 2$ for temperature-driven transitions. Examples are wetting phenomena, polymer desorption, the helix-coil transition in DNA, and unbinding transitions of biomembrane bundles [5], which have gained considerable experimental interest recently [6]. Ensembles of interacting directed lines are also important as the replica formulation of polmers in random media [7]; those in turn are intimately related to theories of nonequilibrium directed growth.

This letter aims at a systematic understanding of delocalization phenomena as renormalized continuum field theories. An exact renormalization group (RG) based on the short-distance algebra of the interaction vertices [8, 9] reveals the existence of a discrete series of universality classes that represent delocalization transitions of a finite number of interacting random walks. The possible existence of analogous 
massless (conformally invariant) field theories is discussed at the end.

One-dimensional quantum particles that interact only via two-body contact forces define the nonlinear Schrödinger model, which is exactly solvable by Bethe ansatz methods (see [12 for a review). This has been applied to unbinding transitions in refs. [10, 11]. In real systems, the interactions are certainly more complicated than the simple pair force of the Schrödinger model. Typically, the force between two lines is screened or enhanced by the presence of further lines. Casimirtype many-body forces (which may be screened at some scale) arise from the coupling of the lines to the surrounding medium, e.g. a correlated fluid [13. There is also experimental evidence for attractive forces between steps on vicinal surfaces. When such interactions are taken into account, the exact solvability is lost, and we are led to study their effect on the delocalization transition by the RG.

Short-ranged multi-particle interactions are easily shown to be irrelevant in the sense of the RG (except 3-body forces for "bosons", see below). Hence weak forces do not alter the asymptotic behavior at large distances, but contribute only corrections to scaling. The new universality classes describe delocalization transitions at finite interaction strength. In a generic field theory, irrelevant vertices are unrenormalizable, i.e. new counterterms are necessary at every order in perturbation theory. Remarkably enough, this proliferation of counterterms does not take place here: the perturbation series remains renormalizable in an $\varepsilon$-expansion although the interaction is irrelevant. This expansion involves analytic continuation in the number $d$ of transversal dimensions, see eq. (雨) below.

In many of the applications above, the lines are effectively impenetrable objects and hence do not intersect. In one dimension, this constraint on their fluctuations is equivalent to the Pauli principle; the particles are fermions. Particles whose trajectories are free to intersect are bosons. Repulsive contact forces suppress intersections and hence generate a crossover from Bose statistics to a low-energy effective Fermi statistics [12]. The RG of this letter offers a unifying view on the interplay between 
dynamics and statistics: delocalization transitions of bosons and fermions fall into the same universality classes, the statistics merely corresponds to parametrizations of the space of interactions about two different fixed points. For the particular case of two- and three-particle interactions, the results are summarized in the RG flow diagram of fig. 1 and the resulting phase diagram of fig. 2. Depending on these interactions, the phase transition can be governed by two distinct fixed points, the free Bose and the "necklace" fixed point, which are discussed in detail below.

We stress that all these fixed points describe ensembles of an arbitrary number of lines; hence the critical exponents do not depend on their number. This result agrees with ref. [10] for the Bose fixed point and is presumably also consistent with the extensive numerical work of ref. [14] if the data are correctly interpreted [15].

Consider a $d$-dimensional system of $p$ massive bosons coupled via forces that decay on some microscopic scale $a$. In the continuum limit $a \rightarrow 0$, the Hamiltonian reads

$$
H_{B}^{(p)}=\frac{1}{2} \sum_{\alpha=1}^{p} \frac{\partial^{2}}{\partial \mathbf{r}_{\alpha}^{2}}+\sum_{m=2}^{p} g_{m} \Phi_{m}^{(p)},
$$

where $\Phi_{2}^{(p)}=\sum_{\alpha<\beta}^{p} \delta^{d}\left(\mathbf{r}_{\alpha}-\mathbf{r}_{\beta}\right), \Phi_{3}^{(p)}=\sum_{\alpha<\beta<\gamma}^{p} \delta^{d}\left(\mathbf{r}_{\alpha}-\mathbf{r}_{\beta}\right) \delta^{d}\left(\mathbf{r}_{\beta}-\mathbf{r}_{\gamma}\right)$, etc. are $m$ particle contact potentials. It describes the universal behavior in the scaling region $\xi_{\perp} \gg a$. In a system with long-ranged forces, (1) is still the correct continuum limit if these forces are irrelevant in the $R G$, i.e. decay with a power of the distance larger than 2. It is convenient to use a description in second quantization,

$$
H_{B}=\int\left(\partial_{\mathbf{r}} \phi^{\dagger}(\mathbf{r}, t)\right)\left(\partial_{\mathbf{r}} \phi(\mathbf{r}, t)\right) \mathrm{d}^{d} \mathbf{r}+\sum_{m \geq 2} g_{m} \Phi_{m}(t),
$$

which is valid for an arbitrary number of lines. The operators $\phi$ and $\phi^{\dagger}$ obey canonical commutation relations and

$$
\Phi_{m}(t)=\frac{1}{m !} \int\left(\phi^{\dagger}(\mathbf{r}, t)\right)^{m}(\phi(\mathbf{r}, t))^{m} \mathrm{~d}^{d} \mathbf{r}
$$

are normal-ordered $m$-particle vertices. With time as the basic scale, these vertices have canonical dimensions $x_{m}=(m-1) d / 2$. Hence the conjugate coupling 
constants $g_{m}$ have dimensions

$$
\varepsilon_{m}=1-x_{m}
$$

The vertices form the short-distance algebra [16]

$$
\begin{aligned}
& \Phi_{k}(t) \Phi_{l}(0)=\sum_{m=\max (k, l)}^{k+l-1} C_{k l}^{m}|t|^{-(k+l-m-1) d / 2} \Phi_{m}(0)+\ldots \\
& C_{k l}^{m}=\frac{m !}{(m-k) !(m-l) !(k+l-m) !}\left(\frac{k+l-m}{2}\right)^{-d / 2} .
\end{aligned}
$$

However, in order to define correlation functions of these vertices, an infrared regularization is necessary. Here the range of each coordinate $r_{i}$ is compactified to a circle of radius $L^{\zeta}$; this regularization preserves translational invariance in space and time. The scale $L$ also serves to define the dimensionless bare couplings $u_{m}=g_{m} L^{\varepsilon_{m}}$ and the dimensionless free energy $F\left(u_{2}, u_{3}, \ldots\right)=L E_{0}\left(g_{2}, g_{3}, \ldots ; L\right)$ in terms of the ground state energy $E_{0}$. The renormalization consists in absorbing the singularities in the perturbation expansion for $F\left(u_{2}, u_{3}, \ldots\right)$ into renormalized couplings $U_{m}$. These singularities are encoded in the operator algebra [8]. By virtue of (5), the beta function $\beta_{m}\left(U_{2}, U_{3}, \ldots\right) \equiv L \partial_{L} U_{m}$ depends only on the $U_{k}$ with $k \leq m$.

Hence consider first the series $F\left(u_{2}\right)=F(0)+\sum_{N=1}^{\infty} F_{N} u_{2}^{N}$, where

$$
F_{N}=L^{1-N \varepsilon_{2}} \frac{(-1)^{N}}{N !} \int\left\langle\Phi_{2}\left(t_{1}\right) \Phi_{2}\left(t_{2}\right) \ldots \Phi_{2}\left(t_{N}\right)\right\rangle_{L} \mathrm{~d} t_{2} \ldots \mathrm{d} t_{N}
$$

and $\langle\ldots\rangle_{L}$ denotes connected expectation values in the unperturbed ground state of an arbitrary particle number sector [17]. (The subsequent manipulations do not depend on the in- and out-states but only on the short-distance structure of the correlation functions.) In the series (7), a single primitive divergence

$$
F_{2}=L^{x_{2}}\left\langle\Phi_{2}\right\rangle_{L} C_{22}^{2} L^{-\varepsilon_{2}} \int_{0}^{L} t^{-1+\varepsilon_{2}} \mathrm{~d} t+O\left(\varepsilon_{2}^{0}\right)=L^{x_{2}}\left\langle\Phi_{2}\right\rangle_{L} \frac{1}{\varepsilon_{2}}+O\left(\varepsilon_{2}^{0}\right)
$$

occurs at $\varepsilon_{2}=0$ (i.e. $d=2$ ). Hence the beta function in minimal subtraction is [18]

$$
\beta_{2}\left(U_{2}\right)=\varepsilon_{2} U_{2}-U_{2}^{2}
$$


This exact renormalizability is intimately related to the summability of the perturbation expansion in the nonlinear Schrödinger model [12]. Generically, (9) would make sense for $\varepsilon_{2}>0$, where $U_{2}$ is relevant at the Gaussian fixed point and generates a crossover to the infrared-stable fixed point $U_{2}^{\star}=\varepsilon_{2}$. As an exact one-loop equation, however, it continues to be valid for $0>\varepsilon_{2}>-1$, where the ultraviolet divergences in $F\left(u_{2}\right)$ can be absorbed in a single counterterm. $U_{2}^{\star}$ is then ultraviolet-stable. In the perturbation series for the correlation functions, singularities analogous to (8) at first order in $u_{2}$ lead to the beta functions

$$
\beta_{m}\left(U_{2}, \ldots, U_{m}\right)=\varepsilon_{m}\left(U_{2}\right) U_{m}+O\left(U_{k} U_{m}\right)
$$

with $(3 \leq k \leq m)$ and

$$
\varepsilon_{m}\left(U_{2}\right)=\varepsilon_{m}-2 C_{m 2}^{m} U_{2}+O\left(U_{2}^{2}\right)
$$

For $m \geq 3$, (11) does not terminate at first order. In $d=1$, however, the combined contribution from higher orders turns out to vanish at the fixed point $U_{2}^{\star}=1 / 2$, so that the infrared dimensions resulting from (11) and (6),

$$
\bar{x}_{m}=1-\varepsilon_{m}\left(U_{2}^{\star}\right)=\frac{m^{2}-1}{2},
$$

are the exact scaling dimensions of the fermionic operators (16) below. The full beta function for $U_{3}$ now follows in a similar way from the singularities in the series $F\left(u_{2}, u_{3}\right)$ at $\varepsilon_{3}=0$. Again, the only primitive singularity occurs at order $u_{3}^{2}$, and hence (with $C_{3} \equiv C_{33}^{3}$ )

$$
\beta_{3}\left(U_{2}, U_{3}\right)=\left(\varepsilon_{3}-3 U_{2}\right) U_{3}-C_{3} U_{3}^{2}
$$

The RG flow of fig. 1 is given by (9) and (13) for $d=1$. In the sequel, we discuss its three fixed points and the implications for the phase diagram.

Free Bosons $\left(U_{2}=U_{3}=0\right)$. The scale-invariant theory is characterized by algebraic finite-size effects $\left\langle\Phi_{m}\right\rangle_{L} \sim L^{-(m-1) / 2}$. For $g_{2}<0$, there is the well-known 
bound state with longitudinal correlation length

$$
\xi_{\|}\left(g_{2}\right) \sim\left|g_{2}\right|^{-2}
$$

and $\left\langle\Phi_{m}\right\rangle_{\infty} \sim\left|g_{2}\right|^{m-1}$, while $g_{2}>0$ generates the crossover to free fermions with (14) now describing the scaling of the crossover length. A repulsive three-particle coupling $g_{3}>0$ is marginally irrelevant. For $g_{2}=0$, it leaves the particles infraredfree, but modifies the theory (e.g. the amplitudes $\left\langle\Phi_{m}\right\rangle_{L}$ ) on scales smaller than

$$
\xi_{\|}\left(g_{3}\right) \sim \exp \left(-1 / g_{3}\right)
$$

for $g_{2} \nearrow 0$, it contributes logarithmic corrections to scaling [15], e.g. $\left\langle\Phi_{m}\right\rangle_{\infty}$ $\sim\left(g_{2} / g_{3} \log \left|g_{2}\right|\right)^{2}$. The marginally relevant $g_{3}<0$ leads to a bound state with (15) and $\left\langle\Phi_{m}\right\rangle_{\infty} \sim \exp \left((m-1) / 2 g_{3}\right)$; the unbinding now takes place on the critical line $g_{2}=g_{2}^{c}\left(g_{3}\right)$ and is governed by the "necklace" fixed point described below.

Free Fermions $\left(U_{2}=\varepsilon_{2}, U_{3}=0\right)$. This fixed point describes the limit $g_{2} \rightarrow \infty$, $g_{3}=0$, where the particles obey the Pauli exclusion principle. Hence the operators $\Phi_{m}$ vanish identically, as follows from the asymptotic crossover scaling of their correlation functions given by (9) and (10), e.g. $\left\langle\Phi_{m}\right\rangle_{L}\left(g_{2}\right) \sim g_{2}^{-m(m-1)} L^{-\left(m^{2}-1\right) / 2}$. Short-ranged interactions are instead described by the fermionic operators

$$
\bar{\Phi}_{m}(t) \equiv \frac{1}{m !} \int \prod_{i=1}^{m} \psi^{\dagger}\left(r+a_{i}\right) \psi\left(r+a_{i}\right) \mathrm{d} r,
$$

where $a_{i}$ are fixed microscopic distances characterizing their range. These operators have scaling dimensions $\bar{x}_{m}=\left(m^{2}-1\right) / 2$ [19] as given by (12) and form an operator algebra of the form (5). Hence for $d=1$, the bosonic Hamiltonian (2) can be written in the equivalent fermionic form

$$
H_{F}=\int\left(\partial_{r} \psi^{\dagger}(r, t)\right)\left(\partial_{r} \psi(r, t)\right) \mathrm{d} r+\sum_{m \geq 2} g_{m} \bar{\Phi}_{m}(t)
$$

The fermionic RG equations are precisely of the form (9), (13) with coefficients $\bar{\varepsilon}_{m}=1-\bar{x}_{m}$ and $\bar{C}_{3}$. Since all interactions (16) are irrelevant, both the bosonic 
fixed point $\left(\bar{U}_{2}=\bar{\varepsilon}_{2}, \bar{U}_{3}=0\right)$ and the necklace fixed point $\left(\bar{U}_{2}=0, \bar{U}_{3}=\bar{\varepsilon}_{3} / \bar{C}_{3}\right)$ are ultraviolet fixed points.

Necklace Theory $\left(U_{2}=\varepsilon_{2}, U_{3}=\bar{\varepsilon}_{3} / C_{3}\right)$. This theory describes the critical transition between the high-temperature phase of free fermions and the "necklace" bound state [20] that forms for $\bar{g}_{3}<\bar{g}_{3}^{c}<0$ and is named after the typical configurations of trajectories shown in fig 2. The transition temperature depends on the parameters $a_{i}$ and is nonuniversal. At this fixed point, the three-particle coupling is relevant. The one-loop RG predicts the exponent $\varepsilon_{3}^{\Delta}=-\bar{\varepsilon}_{3}$ as long as $\bar{\varepsilon}_{3}>-1$. Since $\varepsilon_{3}^{\Delta}$ cannot become $>1$ (this would mean an unphysical divergence of $\left\langle\bar{\Phi}_{3}\right\rangle_{\infty}\left(\bar{g}_{3}\right) \sim \xi_{\|}^{1-\varepsilon_{3}}{ }^{\Delta}$ at the transition) we conclude $\varepsilon_{3}^{\Delta}=1$ for $\bar{\varepsilon}_{3}<-1$. This is confirmed by a mapping of the necklace theory onto a particular point of the critical line of wetting transitions [21, 14]. Hence

$$
\xi_{\|}\left(\bar{g}_{3}\right) \sim\left|\bar{g}_{3}-\bar{g}_{3}^{c}\right|^{-1}
$$

and $\left\langle\bar{\Phi}_{3}\right\rangle_{\infty}\left(\bar{g}_{3}\right)$ approaches a nonuniversal finite value as $\bar{g}_{3} \nearrow \bar{g}_{3}^{c}$. This implies an unusual energy balance for the necklace bound state: its kinetic energy $E_{\text {kin }}$ and potential energy $E_{\text {pot }}$ remain separately finite as the total bound state energy $E_{\text {kin }}+$ $E_{\text {pot }}=-1 / \xi_{\|}$approaches 0 , while at the bosonic transition $E_{\text {kin }} \simeq-E_{\text {pot }} / 2 \simeq 1 / \xi_{\|}$.

From the foregoing RG analysis it transpires that the fixed point $\bar{U}_{3}^{\star}$ is just the first member of a whole family of fermionic necklace theories represented by fixed points $\bar{U}_{m}^{\star}$ of the higher multi-particle interactions $\bar{\Phi}_{m}$. Thus the interplay between attractive and repulsive forces generates a rich scenario of universality classes of interacting directed walks. A detailed understanding of their correlation functions and the various crossover phenomena is within the reach of these RG methods but beyond the scope of this letter. The Bethe ansatz yields the correct asymptotic scaling if and only if the Hamiltonian (2) is in the universality class of the free Bose or Fermi fixed point. Whether analogous methods of exact solution exist for the higher fixed points $\bar{\Phi}_{m}$ is an open question. 
A further interesting question is the existence of analogous fixed points for theories of massless relativistic fermions, where isotropy is restored through particleantiparticle processes. The simplest case is the critical point of the 2D Ising model, a theory of free Majorana fermions with action $S=\int\left(\psi_{+} \partial_{-} \psi_{+}+\psi_{-} \partial_{+} \psi_{-}\right) \mathrm{d}^{2} \mathbf{r}$ in terms of the chiral components $\psi_{+}$and $\psi_{-}$. The lowest-dimensional scalar interaction that is local in the Fermi fields is the irrelevant normal-ordered 4-particle vertex $: \psi_{+} \psi_{+} \psi_{+} \psi_{+} \psi_{-} \psi_{-} \psi_{-} \psi_{-}:=T_{+} T_{-}$(where $T_{+}$and $T_{-}$denote the components of the stress tensor). This interaction is known to be integrable and to generate a crossover whose ultraviolet "necklace" fixed point is the tricritical Ising model 222]. Thus it is tempting to associate the hierarchy of multi-particle interactions with the famous series of minimal conformal field theories [23]. Many other applications involve Dirac fermions with (marginal) local pair interactions. Examples are the ubiquitous Gaussian model with the fermionic action $S=\int\left(\bar{\psi}_{+} \partial_{-} \psi_{+}+\bar{\psi}_{-} \partial_{+} \psi_{-}+g_{2} \bar{\psi}_{+} \psi_{+} \bar{\psi}_{-} \psi_{-}\right) \mathrm{d}^{2} \mathbf{r}$, or the Hubbard model, a theory of two Dirac fermions coupled by similar pair forces (that is relevant to roughening of reconstructed surfaces [24]). In these cases, the effects of the higher interactions (e.g. $T_{+} T_{-}$) are unknown, but since they have a self-coupling in the operator algebra, they are likely to generate similar transitions to massive strong-coupling phases. These multicritical Dirac theories would correspond to conformal field theories with central charge $c>1$.

I am grateful to T.W. Burkhardt, H. Kinzelbach, R. Lipowsky, and R. Netz for useful discussions and comments.

\section{References}

[1] See e.g. J. Frohn et al., Phys. Rev. Lett. 67 (1991), 3543.

[2] M.P.M. den Nijs, in Phase transitions and critical phenomena, vol. 12, ed. C. Domb and J. Lebowitz, Academic, London, 1989.

[3] J. Villain, I. Vilfan, Europhys. Lett. 12 (1990), 523. 
[4] For a recent review, see G. Forgacs, R. Lipowsky, Th.M. Nieuwenhuizen, in Phase transitions and critical phenomena, vol. 13, ed. C. Domb and J. Lebowitz, Academic, London, 1991.

[5] R. Lipowsky, S. Leibler, Phys. Rev. Lett. 56 (1986), 2541.

[6] M. Mutz, W. Helfrich, Phys. Rev. Lett. 62 (1989), 2881.

[7] M. Kardar, Nucl. Phys. B290 (1987), 582.

[8] See M. Lässig and R. Lipowsky, Phys. Rev. Lett. 70 (1993), 1131, and references therein.

[9] M. Lässig and R. Lipowsky, in Fundamental Problems in Statistical Mechanics VIII, ed. H. van Beijeren, North Holland, Amsterdam, 1994.

[10] T.W. Burkhardt, P. Schlottmann, J. Phys. A 26 (1993), L501

[11] C. Hiergeist, M. Lässig, R. Lipowsky, in preparation.

[12] H.B. Thacker, Rev. Mod. Phys. 53 (1981), 253.

[13] H. Li, M. Kardar, Phys. Rev. A 46 (1992), 6490.

[14] R. Netz, R. Lipowsky, Phys. Rev. E 47 (1993), 3039; Phys. Rev. Lett. 71 (1993), 3596; J. Phys. France I, in press.

[15] The authors of ref. [14] report a violation of (14), but their numerical data can probably be accounted for by corrections due to a repulsive 3-particle coupling implicitly present in their system.

[16] The $\Phi_{m}$ are normalized such that $C_{22}^{2}=1$. The terms omited on the r.h.s. contain gradients in space or time or are regular as $\left|t-t^{\prime}\right| \rightarrow 0$.

[17] F. David, B. Duplantier, and E. Guitter, Phys. Rev. Lett. 70 (1993), 2205, have shown that this interaction is renormalizable to all orders in general longitudinal dimensionality.

[18] B. Duplantier, Phys. Rev. Lett. 62 (1989), 2337; J.J. Rajasekaran, S.M. Bhattacharjee, J. Phys. A 24 (1991), L371.

[19] This was first shown in ref. [20] without the explicit use of free fermions. The easiest derivation is to consider the expectation value in a $m$-particle state $\left\langle\chi\left|\bar{\Phi}_{m}\right| \chi\right\rangle_{L}=\int \mid \chi\left(r+a_{1}, \ldots\right.$, $\left.r+a_{m}\right)\left.\right|^{2} \mathrm{~d} r$, which scales as $L^{-(m-1) / 2}\left|\prod_{i<j} \epsilon_{i j}\right|^{2} \sim L^{-\bar{x}_{m}}$ with $\epsilon_{i j} \equiv\left(a_{i}-a_{j}\right) L^{-1 / 2}$, due to the antisymmetry of the fermionic wave function. 
[20] M.E. Fisher, J. Stat. Phys. 34 (667), 1984; M.E. Fisher and D.A. Huse, Phys. Rev. B 29 (1984), 239.

[21] M.E. Fisher, M.P. Gelfand, J. Stat. Phys. 53 (1988), 175.

[22] D.A. Kastor et al., Nucl. Phys. B316 (1989), 590; Al.B. Zamolodchikov, Nucl. Phys. B358 (1991), 524 .

[23] A.A. Belavin et al., Nucl. Phys. B241 (1984), 333.

[24] L. Balents, M. Kardar, Phys. Rev. B 46 (1992), 16031.

\section{Figure Captions}

1. RG flow diagram. $U_{2}, U_{3}$ and $\bar{U}_{2}, \bar{U}_{3}$ denote renormalized two- and threeparticle couplings about the fixed points of free bosons (o) and free fermions $(\bullet)$, respectively. The transition is governed by the Bose fixed point for $U_{3} \geq 0$ and by the necklace fixed point $(\triangle)$ for $U_{3}<0$.

2. Phase diagram in the bare couplings $u_{2}, u_{3}$ of the bosonic theory. Typical world-line configurations: free bosons $\left(u_{2}=u_{3}=0\right)$, free fermions $\left(u_{2} \rightarrow \infty\right.$, $u_{3}=0$ ), bound state for $u_{2}<0$ and $u_{3}>0$, bound state for $u_{2}>0$ and $u_{3}<0$. 